\title{
DanMAX - The new materials science beamline at MAX IV
}

\author{
M. R. V. Jørgensen ${ }^{1,3 *}$, D. R. Sørensen ${ }^{1,3}$, I. Kantor ${ }^{2,3}$, S. F. Oller \\ ${ }^{1}$ Department of Chemistry \& iNANO, Aarhus University, Denmark, \\ ${ }^{2}$ Department of Physics, Technical University of Denmark, Denmark, \\ ${ }^{3}$ MAX IV Laboratory, Lund University, Sweden \\ mads@chem.au.dk
}

The DanMAX beamline [1] located at the diffraction limited storage ring at the MAX IV synchrotron facility [2] and is under commissioning. The beamline is designed to be highly versatile and perform both PXRD and full-field imaging experiments in the energy range $15-35 \mathrm{keV}$. The very brilliant X-ray source (3m IVU16) and a flexible optics system allows for three different band pass modes, $\Delta \mathrm{E} / \mathrm{E} \sim 10^{-4}, 5^{*} 10^{-3} \& 10^{-2}$, and focusing of the beam from $\sim 10 \mu \mathrm{m}$ up to $\sim 1 \mathrm{~mm}$.

DanMAX will have two instruments for PXRD. The first one is equipped with a DECTRIS PILATU3 X 2M CdTe area detector and a silicon drift detector for simultaneous diffraction and X-ray fluorescence spectroscopy. The detector positioning stage will offer large flexibility in both sample to detector distance and in detector tilt to increase the attainable Q range. The instrument is built around a Symétrie Breva hexapod that can accommodate bulky sample environments weighing up to $200 \mathrm{~kg}$. A wide range of sample environments will be available at the beamline. Open standards will be available, both mechanical and software, for fast and easy integration of custom-built sample environments at the beamline. This instrument is expected to be available to users in 2021.

A high resolution instrument will be added in 2022. This instrument will use microstrip detectors and have a large angular coverage. This will enable fast experiment with high resolution. It is planned to start a mail in program for rapid access to this instrument. The instrument will thus be equipped with a robotic sample changer and use computer vision to align the samples, thus ensuring the optimal data quality.

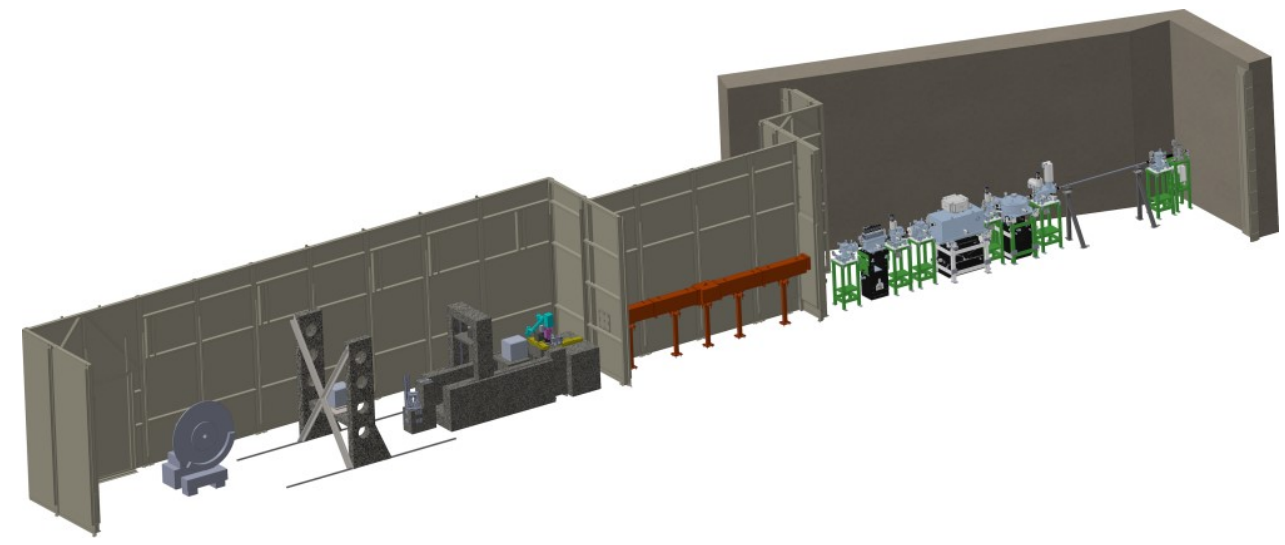

Figure 1. Rendering of the DanMAX beamline. The beam direction is from right to left.

[1] www.maxiv.lu.se/danmax

[2] Tavares, P. F., Leemann, S. C., Sjöström, M. and Anderson, A. J. Synchrotron Rad., 2014, 21, 862-877.

Keywords: in-situ PXRD; Synchrotron beamline; high-resolution PXRD; Total scattering

The funding for the DanMAX project from the Danish Ministry of Higher Education and Science, the Capital Region, the Central Denmark Region, the MAX IV Laboratory, the Technical University of Denmark, University of Copenhagen and Aarhus University is gratefully acknowledged. 